\title{
Progress towards Germ-Line Transformation of the Butterfly Pieris rapae L. (Lepidoptera: Pieridae)
}

Andrew M Stoehr, Xiaoling Tong, Ondrej Podlahas and Antónia Monteiro*

Department of Ecology and Evolutionary Biology, Yale University, New Haven, CT 06511, USA

\begin{abstract}
Germ line transformation is a powerful research tool to probe and manipulate gene function but currently the number of insect species transformed is small and biased towards Diptera. Here we develop transgenic techniques for a butterfly in the family Pieridae, the widely-studied cabbage white butterfly (Pieris rapae L; Lepidoptera: Pieridae). Germ line transformation of pierids is useful because butterfly wing patterns have become model systems in evolutionary developmental biology, and the previously transformed butterflies are members of a different family, the nymphalids. We used a piggyBac[3xP3-EGFP] construct as a marker for germ line transformation and obtained a single transformed adult that did not reproduce but which displayed fluorescence in its eyes. After identifying the genomic insertion site for the construct we conclude that this individual was likely transgenic. However, short of longer-term inheritance data, we discuss two less likely alternative possibilities for our data.
\end{abstract}

Keywords: Pieris rapae; PiggyBac; Lepidoptera; TAIL-PCR

\section{Introduction}

Butterfly wing patterns are becoming important model systems for addressing questions in evolutionary developmental biology (evo-devo) [1-3], however, to examine the evolution of gene function or regulation in butterflies it will be important to perform germ-line transformation in several of these insects.

Two species of butterfly, Bicyclus anynana and Danaus plexippus have been genetically transformed using piggyBac transposable elements and zinc-finger nucleases [4,5], respectively. Both of these species, however, belong to the same family (Nymphalidae), so members of other families currently lack a close functional genetic model.

Here, we report on efforts to transform the germ-line of the butterfly Pieris rapae belonging to the family Pieridae. P. rapae is easily reared and is a useful insect for research in evo-devo as well as in other fields, including physiology [6], insect-host plant interactions [7], and other aspects of ecology and behavior [8]. Indeed, P. rapae is probably among the most widely studied butterflies in addition to being an important agricultural pest [9]. Given that the use of transgenic insects is increasingly viewed as having potential for important applications in pest control [10], the development of transgenic techniques for this species may eventually help in its control.

In germ-line transformation experiments that try to introduce a foreign piece of DNA into a host species in order to test its function, there are a series of confirmatory steps required to show successful genome integration. First, the transgenic individual should display a visible marker of transgenesis. This is often accomplished by the insertion of a marker cassette that includes a fluorescent protein gene driven by a tissue-specific promoter. Second, inserted DNA should be PCR-amplified from the genome of the transgenic individual. Third, the genomic sequences flanking the inserted DNA should be different from those flanking it when still in the plasmid vector. Fourth, the marker gene should be inherited across multiple generations.

Here we obtained data for the first three of the four confirmatory steps described above. We used a piggyBac construct containing the [3xP3-EGFP] marker cassette that upon insertion into the germ line led to green fluorescence in the eyes of Pieris rapae. We amplified the EGFP gene from the genome of a transgenic individual, and obtained flanking genomic data for the inserted sequence. Due to premature death of the individual we were not able to confirm successful inheritance of the transgene.
Finally, we report on the use of a simple method, thermal asymmetric inter-laced PCR, i.e. TAIL-PCR [11] for identifying the flanking regions of the insert in both $P$. rapae and in another previously transformed butterfly, B. anynana.

\section{Materials and Methods}

\section{Butterfly husbandry}

P. rapae larvae were reared on collard greens inside a climate chamber at $23^{\circ} \mathrm{C}, 70 \% \mathrm{RH}$, and $16 \mathrm{~h}$ light: $8 \mathrm{~h}$ dark light cycle. Pupae were placed inside cylindrical mesh cages $(30 \mathrm{~cm}$ diameter, $40 \mathrm{~cm}$ height) where adults emerged and mated. Adults were fed on artificial nectar (honey, water, and yellow dye solution) and induced to lay eggs on strips of Parafilm. To do this, a water-filled container was covered with a section of cabbage leaf and a strip of Parafilm was wrapped around the outside of the container (Figure 1A). Female butterflies attracted to the cabbage leaf would then "mistakenly" lay eggs on the Parafilm [12]

\section{Embryo injections}

Injection procedures essentially followed that of Marcus et al. [5]. Within 2 hours of egg laying, eggs were injected, while still attached to the Parafilm, with a mixture of two plasmids at equal concentrations, $(600 \mathrm{ng} / \mu \mathrm{l})$, one plasmid carrying the desired insert (piggyBac construct piggyBac[3xP3 - EGFP, Hsp70-spalt]) and the other a helper plasmid carrying the transposase sequence (plasmid construct pHsp82PBac) [13]. The piggyBac construct carried a transformation marker - the EGFP gene under the control of the synthetic $3 \mathrm{xP} 3$ promoter that drives gene expression in the eyes [14,15], as well as a Drosophila melanogaster spalt sequence, under the control of the D. melanogaster Hsp70 promotor, because our ultimate goal was to perform functional

*Corresponding author: Antónia Monteiro, Department of Ecology and Evolutionary Biology, Yale University, New Haven, CT 06511, USA, Tel: 659755 1591; E-mail: antonia.monteiro@nus.edu.sg

Received June 12, 2015; Accepted July 10, 2015; Published Jull 13, 2015

Citation: Stoehr AM, Tong X, Podlahas O, Monteiro A (2015) Progress towards Germ-Line Transformation of the Butterfly Pieris rapae L. (Lepidoptera: Pieridae). Gene Technology 4: 123. doi: 10.4172/2329-6682.S1-001

Copyright: @ 2015 Stoehr AM, et al. This is an open-access article distributed under the terms of the Creative Commons Attribution License, which permits unrestricted use, distribution, and reproduction in any medium, provided the original author and source are credited. 

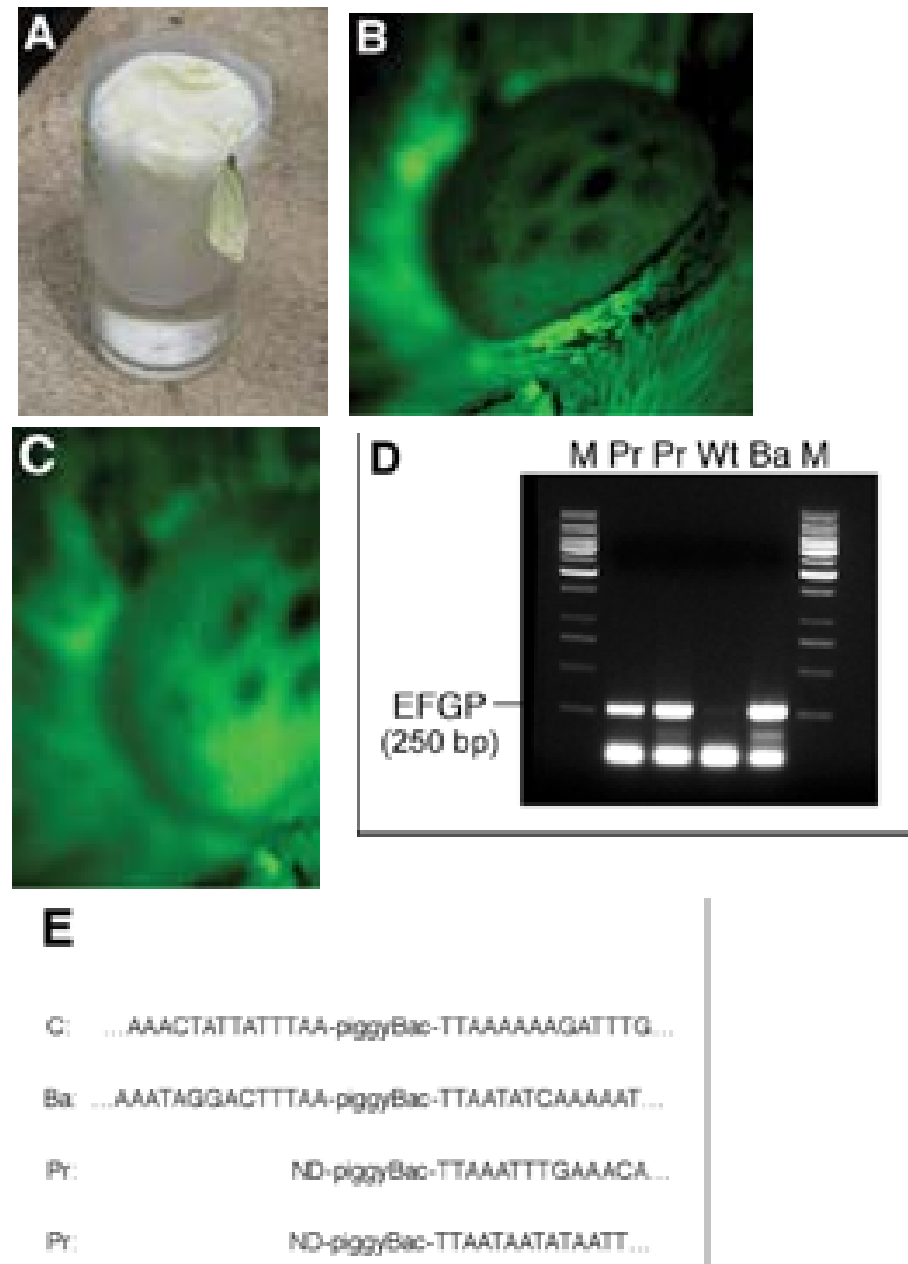

Figure 1: Steps towards the germ-line transformation of Pieris rapae. (A) Female $P$. rapae laying eggs on Parafilm. Wild-type (B) and EGFP-expressing (C) P. rapae eyes photographed under similar blue-light excitation conditions and enhanced for brightness and contrast, (D) PCR amplicons of EGFP ( 250 bp) from genomic DNA of the $\mathrm{P}$. rapae putative transgenic (Pr, two lanes), $\mathrm{P}$. rapae wildtype $(\mathrm{Wt})$ and a $\mathrm{B}$. anynana $(\mathrm{Ba})$ transgenic line. M is the DNA ladder, (E) Partial sequences flanking the piggyBac insertion sites. Target site (TTAA) is underlined. (Complete sequences are given in Supp. File 1). Left of target site is the left arm of piggyBac, right is the genomic sequence of $\mathrm{P}$. rapae $(\mathrm{Pr})$ (two insertions) or $\mathrm{B}$. anynana $(\mathrm{Ba})$. C (control) is the sequence expected if the vector had been inherited extra-chromosomally. ND ("No Data" refers to the fact that we could not amplify that side of the insert in Pieris.

assays of the role of spalt in $P$. rapae wing color patterning. To inject eggs, we used a pulled glass needle and a Picospritzer III microinjector. Following injection, we placed the Parafilm strips with eggs inside plastic Petri dishes containing some moistened cotton, and these dishes were then placed in an incubator at $27^{\circ} \mathrm{C}$ and $80 \%$ relative humidity. Eggs were checked daily for hatching and larvae were transferred to host plants (collards) with a small paintbrush. These larvae were reared to adulthood.

\section{Crosses and screening for transformants}

Injected adults were crossed with wild type individuals of the opposite sex to produce a second generation. Because of space limitations, and because the butterflies seemed to mate and thrive better in cages with multiple butterflies, these crosses were carried out in several cages, each containing 8-15 butterflies. Offspring from these crosses were reared to adulthood and then screened for the presence of the EGFP protein in the eyes. To screen for EGFP expression, we photographed the eyes of all potentially positive butterflies as well as several known wild types (to serve as controls) using a Zeiss AxioCam Mrc camera attached to a Zeiss Stereo Discovery V8 microscope with an X-cite 120 XL light source and a GFP band pass filter (Zeiss Cat. No. 9108384116). We then created digital contact sheets (using the Automate/Contact Sheet II function of Adobe Photoshop CS3) containing the images of all butterfly eyes photographed that day, and adjusted the brightness and contrast of these contact sheets until the settings best distinguished among individuals (Figure $1 \mathrm{~B}$ and $1 \mathrm{C}$ ). The fifteen butterflies with the brightest eyes were selected and then crossed with wild type individuals, with the intent to produce a generation to use for heat shocks to test gene function (but see above). When the selected butterflies died, we extracted genomic DNA to confirm the presence of the EGFP sequence through PCR. We used the following forward and reverse primers: EGFP forward primer 5'-ACGACGGCAACTACAAGACCC-3'; EGFP reverse primer 5'-GTGTTCTGCTGGTAGTGGTCG-3'; and the following PCR amplification conditions: An initial denaturing step of $94^{\circ} \mathrm{C}$ for $4 \mathrm{~min}$, followed by 45 cycles of $94^{\circ} \mathrm{C}$ for $45 \mathrm{sec}, 59^{\circ} \mathrm{C}$ for 30 sec, and $72^{\circ} \mathrm{C}$ for $50 \mathrm{sec}$, followed by a final elongation step of $72^{\circ} \mathrm{C}$ for $6 \mathrm{~min}$ and $30 \mathrm{sec}$. 


\section{Obtaining flanking genomic sequences to the piggyBac insertion}

We used TAIL-PCR [11] to isolate the left and right genomic sequences flanking the piggyBac insertion in individuals that were positive for EGFP. TAIL-PCR uses a set of nested, high-melting-temperature primers derived from the ends of the transposon, along with lower-melting-temperature degenerate primers that bind to many possible flanking sequences, in three successive rounds of PCR. The first and second rounds of PCR use alternating high- and low-temperature annealing cycles to selectively amplify those products that include the insertion site, with the second and third rounds using a dilution of the first-round (1:20) and second-round (1:10) products as the template, respectively. The third round of PCR uses normal thermocycling conditions. The three nested specific primers for the left side of the piggyBac vector were 5'-CATTTTGACTCACGCGGTCGTTATAGTTC-3' (L1, for primary round of PCR), 5'-CAGTGACACTTACCGCATTGACAAGCA-3' (L2, secondary round), 5'-CGACTGAGATGTCCTAAATGCACAG-3' (L3, tertiary round). The right side specific primers were 5'-CCAAATGAAGTGCCTGGTACATCAG-3' (R1), 5'-GTGCCAAAGTTGTTTCTGACTGACTAATAAG-3' (R2), and 5'-TCGATATACAGACCGATAAAACACATGCGTC-3' (R3). The six degenerate primers used were 5'-NGTCGASWGANAWGAA-3' (AD1), 5'-TGWGNAGSANCASAGA-3'(AD2), 5'-AGWGNAGWANCAWAGG-3' (AD3), 5'-STTGNTASTNCTNTGC-3' (AD4), 5'-NTCGASTWTSGWGTT - 3' (AD-5), 5'-WGTGNAGWANCANAGA 3'(AD-6). Cycling conditions for each round of PCR followed those of Wang et al. (2007). After the primary round of PCR, a 1:20 dilution of the PCR product was used as the template for the secondary round of PCR. A 1:10 dilution of the secondary product was used as the template for the tertiary round of PCR. When these conditions failed to amplify the sequence to the right of the insertion site, we used a new combination of right side specific primers: 5'-GTATACCATCTTAGCTGGCTTCGG-3' (R1), 5'-CTTATTAGTCAGTCAGAAACAACTTTGGCAC-3'(R2), and 5'-GACGCATGTGTTTTATCGGTCTGTATATCGA-3' (R3). Tertiary PCR products, when present, were run on a gel, cut out, purified using the QIAquick Gel Extraction Kit (QIAGEN Sciences, Maryland, USA) and sequenced.

\section{Results and Discussion}

Transposable element is likely capable of transforming Pieris rapae, but that improvements in the methods, particularly the development of better We injected 1,515 P. rapae eggs with a piggyBac[3xP3-EGFP, Hsp70-spalt] construct, and helper plasmid ( $\mathrm{pHsp} 82 \mathrm{Pbac}$ ), reared the 75 survivors ( 34 females, 41 males) to adulthood and crossed these with wild-type butterflies to create a screening generation ( $472 \mathrm{females}$, 535 males). Adults of this generation were screened for the presence of green fluorescence in the eyes above normal auto-fluorescence levels seen in wildtype butterflies (Figure $1 \mathrm{~B}$ and $1 \mathrm{C}$ ), and putative positives (8 females, 7 males) were tested for the presence of the EGFP sequence using PCR. One butterfly produced a PCR product of the predicted size, that when sequenced was confirmed to be EGFP (Figure 1D). Because a single individual was confirmed to carry the EGFP gene this indicated that fluorescence levels derived from EGFP were of a similar magnitude as bright auto-fluorescent wild type eyes. Because we performed group crosses we cannot be sure that every parent mated and produced offspring, so a minimal estimate of transformation efficiency in this experiment is at least 1 parent, out of 75 , produced transgenic offspring, or $1.3 \%$. The real rate is likely to be higher.

Sometimes plasmids are inherited extra-chromosomally, i.e. without ever inserting into the genome [14]. Thus, to confirm that the piggyBac transposase successfully catalyzed the insertion of the piggyBac vector, we amplified and sequenced the flanking regions of the genomic insertion site using TAIL-PCR. To test the broader applicability of the TAIL-PCR method, we also performed the procedure on a putative transgenic B. anynana butterfly known to carry the EGFP sequence flanked by piggyBac arms.

For the P. rapae individual we estimated the presence of at least two independent piggyBac insertion sites by successfully amplifying $P$. rapae genomic sequences flanking the left arm of piggyBac. Two degenerate primers (AD1 and AD6) identified the same insert, whereas the second insert was the result of priming with AD2 (Figure 1E). This procedure suggests at least two insertion sites. We successfully amplified genomic sequences flanking both sides of piggyBac (using AD6 for the left side, $\mathrm{AD} 2$ for the right) for the B. anynana butterfly as well (Figure 1E). For reasons we have yet to identify, we were unable to produce the desired products using the piggyBac right arm primer sets for $P$. rapae. The sequences of the left side products, however, show the characteristic piggyBac transposase insertion sequence (TTAA) and also do not match the sequence that flanks piggyBac in the injected plasmid (Figure 1E).

We propose that this individual was likely a complete transgenic; however two less plausible alternatives can also be considered that would make this individual only partly transgenic, e.g, a mosaic. For instance, there remains a slight possibility that the two original plasmids were inherited extra-chromosomally and that the integration event took place in the G1 individual, not its parent, making this individual a mosaic and not a true transgenic. This mechanism, while plausible, is in our opinion unlikely because we found no evidence for the presence of the original plasmid flanking sequence in the genome of this G1 individual and because the eyes of this individual were homogeneously bright green. It is more likely that the plasmid was not inherited extra-chromosomally but was inherited already integrated into the germ-cells of the injected parent, making the G1 individual a true transgenic. A second possibility involves the integration event having taken place in the genome of a vertically inherited symbiont, and not in the actual butterfly's genome, although we have no knowledge of this type of mechanism ever happening in similar experiments. Continued inheritance of the EGFP sequence over several generations, and clear Mendelian patterns of segregation would support the butterfly transgenic explanation over the other two. This study therefore falls short of definitively concluding that the germ-line of $\mathrm{P}$. rapae was transformed with piggyBac. PiggyBac, however, has been successfully used to transform the germ-line of another butterfly, the nymphalid $B$. anynana [15-17], and three other Lepidopterans: the moths, Bombyx mori [18], Pectinophora gossypiella [19] and Cydia pomonella [20]. It is likely, thus, that piggyBac can successfully transform $P$. rapae.

Finally, we have amplified the insertion site flanking regions for transformed $P$. rapae and B. anynana butterflies utilizing TAIL-PCR, a method that can have some advantages over other commonly used methods (e.g. inverse PCR and southern blotting) used to identify flanking regions. Unlike some of these other methods that require larger quantities of high quality DNA (southern blotting), and enzyme restriction and ligation reactions (inverse PCR), TAIL-PCR requires only standard PCR techniques with a set of nested specific primers and degenerate primers.

Although these early results for $P$. rapae are encouraging, we see room for improvement. Particularly valuable would be the development of better transformation markers. Wild-type $P$. rapae eyes are considerably auto-fluorescent under blue-light excitation (much more so, than, for example B. anynana; contrast (Figure $2 \mathrm{~A}$ with $2 \mathrm{C}$ ), making it difficult 


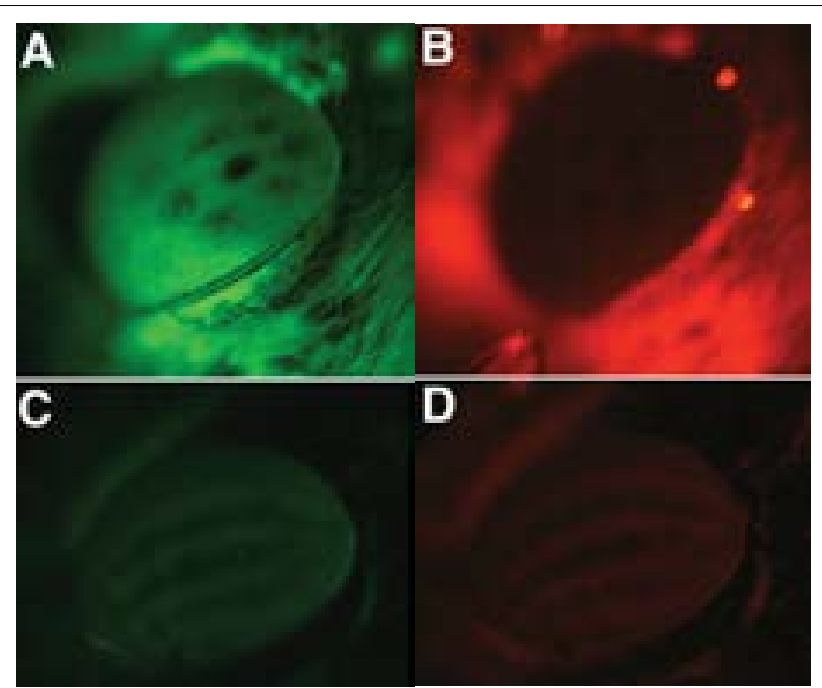

Figure 2: Autofluorescence in Pieris rapae and Bicyclus anynana. (Figure 2A with 2C) Under similar blue-light excitation conditions, $P$. rapae (A) is considerably more autofluorescent than $B$. anynana $(\mathrm{C})$. (Figure $2 \mathrm{~B}$ with $2 \mathrm{C}$ ) Under similar green-light excitation conditions, $P$. rapae (B) is less autofluorescent than $B$. anynana (D) (The very bright fluorescence in $(B)$ comes from scales surrounding the eye; the eye is the very dark oval-shaped portion.) All photos taken using the same exposure time.

to distinguish weakly EGFP-fluorescing individuals from wild type. Future tests of alternative markers, such as DsRed, might facilitate this goal [15] because $P$. rapae is less auto-fluorescent under greenlight excitation (Figure 2B vs. 2D). This might allow earlier screening, perhaps at the early larval instars or even the embryonic stage [21]. We also do not know why our TAIL-PCR procedure successfully amplified both sides of the piggyBac insert in B. anynana but only one side in $P$. rapae. The most likely explanation, perhaps, is that the combination of random primers simply could not work together with the specific primers for the particular genomic location of this insertion. If the insertions were close to the ends of chromosomes this would prevent adequate priming sites on the telomere side. An alternative explanation is a mutation in the right-arm of the piggyBac sequence preventing correct priming.

It was unfortunate that our transgenic P. rapae individual died before reproducing, preventing us from establishing a multi-generational line to determine the inheritance pattern and stability of the piggyBac vector in this species. $P$. rapae is a hardy species in captivity, but in any given generation some individuals die, particularly if they do not quickly learn to feed on the artificial nectar sources we provide. It is also possible that transgenesis contributed to the death of the single recovered butterfly. Our previous experience with B. anynana, however, shows that transgenic butterflies can live normal lives $[5,17,19,22]$.

Finally, we recently showed that the $3 x P 3-E G F P$ marker cassette does not lead to stable green fluorescence in the eyes of transgenic B. anynana [23]. This is most likely because the transcription factor eyeless that binds $3 \mathrm{xP} 3$, stops being expressed in eyes shortly after adult emergence [23], eliminating expression of EGFP, and making transgenic adults look similar to wild type adults. This work suggests that future transgenic experiments with P. rapae and other butterflies should undertake screening for transgenics during the larval and pupal stages to increase the likelihood of detecting a transgenic animal.

In conclusion, we have shown that the piggyBac transformation markers, should facilitate not only the functional testing of candidate genes in wing pattern development in this species - our ultimate goal but also the use of $P$. rapae, a widely-studied organism, in other contexts that require transgenic tools.

\section{References}

1. Beldade P, Brakefield PM (2002) The genetics and evo-devo of butterfly wing patterns. Nat Rev Genet 3: 442-452.

2. McMillan WO, Monteiro A, Kapan DD (2002) Development and evolution on the wing. Trends Ecol Evol 17: 125-33.

3. Ramos DM, Monteiro A (2007) Transgenic approaches to study wing color pattern development in Lepidoptera. Mol Biosyst 3: 530-535.

4. Merlin C, Beaver LE, Taylor OR, Wolfe SA, Reppert SM, et al. (2013) Efficient targeted mutagenesis in the monarch butterfly using zinc-finger nucleases. Genome Res 23: 159-168.

5. Marcus JM, Ramos DM, Monteiro A (2004) Germline transformation of the butterfly Bicyclus anynana. Proc Biol Sci 271 Suppl 5: S263-265.

6. Arikawa K, Wakakuwa M, Qu X, Kurasawa M, Stavenga DG et al. (2005) Sexual dimorphism of short-wavelength photoreceptors in the small white butterfly, Pieris rapae crucivora. J Neurosci 25: 5935-5942.

7. Mumm R, Burow M, Bukovinszkine'Kiss G, Kazantzidou E, Wittstock U, et al. (2008) Formation of Simple Nitriles upon Glucosinolate Hydrolysis Affects Direct and Indirect Defense Against the Specialist Herbivore, Pieris rapae. Journal of Chemical Ecology 34: 1311-1321.

8. Obara Y, Ozawa G, Fukano Y, Watanabe K, Satoh T, et al. (2008) Mate preference in males of the cabbage butterfly, Pieris rapae crucivora, changes seasonally with the change in female UV color. Zoolog Sci 25: 1-5.

9. Hill DS (1987) Agricultural Insect Pests of Temperate Regions and Their Control. Cambridge University Press, New York.

10. Hoy MA (2000) Transgenic arthropods for pest management programs: risks and realities. Exp Appl Acarol 24: 463-495.

11. Liu YG, Whittier RF (1995) Thermal asymmetric interlaced PCR: automatable amplification and sequencing of insert end fragments from $\mathrm{P} 1$ and $\mathrm{YAC}$ clones for chromosome walking. Genomics 25: 674-681.

12. Webb SE, Shelton AM (1988) Laboratory rearing of the imported cabbageworm New York's Food and Life Sciences Bulletin 122: 1-6.

13. Horn C, Schmid BG, Pogoda FS, Wimmer EA(2002) Fluorescent transformation markers for insect transgenesis. Insect Biochem Mol Biol 32: 1221-1235.

14. Robinson KO, Ferguson HJ, Cobey S, Vaessin H, Smith BH (2000) Spermmediated transformation of the honey bee, Apis mellifera. Insect Mol Biol 9: 625-634.

15. Monteiro A, Chen B, Ramos DM, Oliver JC, Tong X, et al. (2013) Distal-less regulates eyespot patterns and melanization in Bicyclus butterflies. J Exp Zoo B Mol Dev Evol 320: 321-331. 
Citation: Stoehr AM, Tong X, Podlahas O, Monteiro A (2015) Progress towards Germ-Line Transformation of the Butterfly Pieris rapae L. (Lepidoptera: Pieridae). Gene Technology 4: 123. doi: 10.4172/2329-6682.S1-001

16. Marcus J, Ramos D, Monteiro A (2004) Germline transformation of the butterfly Bicyclus anynana. Proceedings of the Royal Society B-Biological Sciences 271: S263-S5

17. Tong X, Hrycaj S, Podlaha O, Popadic A, Monteiro A (2014) Over-expression of Ultrabithorax alters embryonic body plan and wing patterns in the butterfly Bicyclus anynana. Dev Biol 394: 357-366

18. Tamura T, Thibert C, Royer C, Kanda T, Abraham E, et al. (2000) Germline transformation of the silkworm Bombyx mori L. using a piggyBac transposonderived vector. Nat Biotechnol 18: 81-84.

19. Peloquin JJ, Thibault ST, Staten R, Miller TA (2000) Germ-line transformation of pink bollworm (Lepidoptera: gelechiidae) mediated by the piggyBac transposable element. Insect Mol Biol 9: 323-333.
20. Ferguson $H J$, Neven LG, Thibault ST, Mohammed A, Fraser M (2011) Genetic transformation of the codling moth, Cydia pomonella L., with piggyBac EGFP. Transgenic Res 20: 201-214.

21. Thomas JL, Da Rocha M, Besse A, Mauchamp B, Chavancy G (2002) 3xP3 EGFP marker facilitates screening for transgenic silkworm Bombyx mori L. from the embryonic stage onwards. Insect Biochem Mol Biol 32: 247-253.

22. Ramos DM, Kamal F, Wimmer EA, Cartwright AN, Monteiro A (2006) Tempora and spatial control of transgene expression using laser induction of the hsp70 promoter. BMC Dev Biol 6: 55.

23. Das Gupta M, Chan SK, Monteiro A (2015) Natural Loss of eyeless/Pax6 Expression in Eyes of Bicyclus anynana Adult Butterflies Likely Leads to Exponential Decrease of Eye Fluorescence in Transgenics. PLoS One 10 e0132882. 